Cahiers de recherches médiévales

Journal of medieval studies

$1 \mid 1996$

Croisades et idée de croisade à la fin du Moyen Âge

\title{
Le concile de Ferrare et la croisade
}

\section{Franco Cardini}

\section{OpenEdition}

Journals

Édition électronique

URL : https://journals.openedition.org/crm/2513

DOI : $10.4000 / \mathrm{crm} .2513$

ISSN : 1955-2424

Éditeur

Honoré Champion

Édition imprimée

Date de publication : 15 janvier 1996

Pagination : 45-52

ISSN : 1272-9752

\section{Référence électronique}

Franco Cardini, «Le concile de Ferrare et la croisade », Cahiers de recherches médiévales [En ligne], 1 |

1996, mis en ligne le 05 février 2008, consulté le 15 décembre 2022. URL : http://

journals.openedition.org/crm/2513; DOI : https://doi.org/10.4000/crm.2513 


\section{Le concile de Ferrare et la croisade}

La causa fidei, et par conséquent le negotium crucis, avaient été, avec la causa unionis et la causa reformationis, les principales préoccupations des grandes assises de l'Église jusqu'aux deux grands conciles de Lyon du XIIr siècle. Après l'utilisation que le pape d'Avignon - en ces temps où l'outil du concile n'avait pas la parole - avait fait de la croisade en deçà et au-delà de la mer comme arme de pression politique et fiscale', tant au concile de Constance qu'à celui de Bâle on voyait progressivement s'affirmer une tendance de la Curie pontificale, du concile lui-même et de ses souverains laïcs à gérer ensemble les croisades. Cette tendance, conforme aux principes chers au mouvement conciliaire, s'exprimait contre les turcs mais aussi contre les ennemis internes de la Chrétienté - comme les hussites en Bohême - par la formation de «Ligues saintes» dont le pontife avait certes la présidence mais dont le mécanisme politique et militaire ne pouvait être mis en route qu'avec le consensus des princes et des prélats assistant aux assises conciliaires ainsi que des cardinaux qui composaient le Sacré Collège ; or ceux-ci - les prélats comme les cardinaux - se révélaient généralement être des voix qui se rangeaient à l'avis de leurs princes respectifs aux faveurs desquels ils devaient souvent leur rang dans l'Église. Ainsi révolue l'époque de la Chrétienté conçue comme un corps unique avec deux chefs - le pape in spiritualibus, l'empereur in temporalibus -, on voyait petit à petit affleurer un nouveau modèle pour ainsi dire «fédéral», dont la croisade était le banc d'essai.

Après le désastre de la bataille de Nicopolis en 1396 qui semblait avoir infligé un coup irréparable à l'idée même de croisade, la météore de Tamerland avait traversé les cieux euro-asiatiques en rallumant - et bien que Tamerland soit lui-même musulman - de nouveaux espoirs et en proposant à nouveau les vieux rêves d'alliance entre tartares et chrétiens contre l'Islam, ces mêmes rêves qui avaient, depuis la moitié du XIII siècle, rempli les pages des auteurs de traités d'une croisade qui ne s'était jamais faite. Il n'en est pas moins vrai qu'à partir de la bataille d'Ankara en 1402, au sortir de laquelle la puissance et le prestige ottomans étaient affaiblis et compromis, le danger pour le moins immédiat d'une conquête de la capitale de l'empire byzantin de la part des turcs paraissait s'être éloigné; et, avec lui, les appels des grecs à une croisade occidentale s'étaient faits moins pressants, et les offres de mettre fin au schisme d'Orient en échange d'une aide militaire étaient par conséquent moins explicites et moins ardentes que celles d'aide de la Chrétienté latine. Par une sorte de paradoxe ironique et dramatique, la tension pour la croisade diminuait en Occident - un peu en conséquence de la raclée encourue en 1396, un peu comme résultat de la diminution de la pression ottomane sur Constantinople juste au moment où une éventuelle offensive contre les effectifs ottomans affaiblis

${ }^{1}$ Cf. N. Housley, The Avignon papacy and the crusades 1305-1378, Oxford, 1986.

Cahiers de Recherches Médiévales (XIII-XV s.), 1, 1996 


\section{Franco CARDINI}

aurait eu de meilleures probabilités de succès. Mais la persistance et aussi la complication du schisme occidental des premières années du XIII siècle ainsi que les questions européennes en général (guerre de Cent ans, luttes pour la succession au trône impérial romain-germanique, expansionnisme des Angevins et de Durazzo à partir de Naples vers l'Italie centrale et la région des Balkans et du Danube, confrontation entre Venise et Milan et entre Milan et Florence en Italie, crise hussite en Bohême) rendaient vaine toute perspective d'action en faveur d'une croisade commune, tandis qu'entre la Mer Noire et celle du Levant l'inimitié entre Venise et Gênes se manifestait en une série d'épisodes, y compris belliqueux, où les deux puissances opposées ne dédaignaient pas d'avoir recours l'une contre l'autre à des appuis islamiques: au sultanat ottoman de Brusse les gênois, à celui - mamelouk du Caire les vénitiens.

En 1413, le sultan ottoman Mahomet I avait eu le dessus sur ses frères - qui étaient aussi ses rivaux pour le trône - grâce à l'appui du basileus byzantin Manuel Paléologue : raison pour laquelle il était resté toute sa vie fidèle au souverain grec. Les choses changèrent avec le sultan Murat, fils de Mahomet I, car il faut aussi dire que Manuel avait favorisé un de ses parents dans la lutte pour la succession au trône; et Murat, vainqueur de l'opposition familiale, avait riposté en assiégeant Constantinople entre juin et septembre $1422^{2}$. Le siège prit fin sans que cela ait changé quoi que ce soit, mais c'était signe que la brève période de trêve accordée aux grecs après la journée d'Ankara était terminée, et que reprenait la lente agonie de l'empire - désormais réduit, à peu de chose près, à sa seule capitale. En janvier 1421. Manuel avait entre-temps élevé son fils Jean VIII, au trône en tant que coempereur et, à la mort de son père en 1425 , celui-ci devint seul et unique basileus et gouverna les restes de l'empire en collaboration avec ses frères qui eux, gouvernaient - pratiquement sans dépendre de lui - à Thessalonique et au Péloponnèse. La situation apparaissait désormais si grave qu'au cours de l'été 1423, Andronic Paléologue (un des fils de Manuel et frère de Jean) avait en tout cas cédé Thessalonique aux vénitiens, choix qui avait ultérieurement irrité le sultan Murat déjà mal disposé à l'égard des Paléologues et décidé à en précipiter la fin. Alors que la Morée, sous la conduite des despotes Théodore, Constantin et Thomas, frères de l'empereur, semblait en mesure de soutenir à elle seule la menace turque, Jean estimait de son côté que désormais, seul l'appui de l'Occident aurait sauvé Byzance; et il décida de s'adresser pour cela aux frères latins dans le Christ qu'il n'avait à vrai dire jamais trop aimés en leur offrant - et surtout au pape - le seul enjeu appétissant dont il disposait et qu'ils paraissaient ambitionner : l'union des Églises.

Ce choix ne fut pour lui ni facile ni automatique. En 1422 déjà, quand son père Manuel - très sceptique sur la possibilité de voir le schisme se terminer et même à la fin contraire aux expériences en ce sens - était encore en vie, Jean avait visité certaines contrées occidentales - Venise, Milan, la Hongrie - et avait été bénévolement accueilli par l'empereur Sigismond. Francesco Filelfo, un jeune savant des Marches émigré depuis peu à Constantinople, lui servait d'interprète. Mais de toutes les puissances occidentales - à part Sigismond, pour qui le problème des croisades se limi-

\footnotetext{
${ }^{2}$ Cf. G. Orstrogorsky, Storia dell'impero bizantino, tr. it., Torino, 1966 ; F. Babinger, Maometto il Conquistatore, tr. it., Torino, 1967.
} 
tait à contenir la menace turque dans la région des Balkans et du Danube qui le concernait de plus près - le seul sincèrement disposé à entreprendre une politique de longue haleine contre les ottomans semblait être Philippe le Bon, le duc de Bourgogne, qui en 1421 avait déjà (au service formel de Henri VI d'Angleterre et du duc de Bedford régent de la France en son nom) envoyé en Orient un «observateur», Gilbert de Lannoy; le «Grand Duc d'Occident» renouvellera cette expérience en 1432 en envoyant en Terre Sainte et en Asie Mineure un homme qui avait toute sa confiance, Bertrandon de la Brocquière, lequel rassemblera dans un beau rapport, un quart de siècle plus tard, les résultats de son expérience. Mais la nature des forces en jeu n'avait pas tardé à révéler sa nature authentique: les grecs auraient désiré une aide militaire forte et rapide en échange d'une union des Églises lui faisant suite; la Curie romaine prétendait à une union immédiate comme prix pour entreprendre un projet de croisade, même s'il devait être effectué rapidement ; de leur côté, les puissances occidentales étaient très tièdement disposées à s'engager dans une entreprise qui aurait comporté des coûts élevés, des risques multiples et dont les bénéfices en termes de prestige et de pouvoir seraient revenus uniquement au pape; et dans l'Église elle-même les forces conciliaires - d'ailleurs étroitement liées aux princes laïcs d'Europe - considéraient la réforme entreprise avec le concile de Constance comme objectif prioritaire du moment et jugeaient la croisade comme un but secondaire et subordonné à l'autre. Entre temps, à Constantinople - et Jean le savait bien - une partie considérable de l'opinion publique, aussi bien ecclésiastique que laïque, commençait à se demander si la subordination à Rome était vraiment un mal mineur par rapport à la chute aux mains des turcs. En d'autres termes, avant et plutôt qu'une extrema ratio, le dramatique voyage en Occident de l'héritier au trône qui deviendrait ensuite l'empereur Jean apparaissait comme un choix de camp qui lui procurerait de fiers partisans et des adversaires acharnés. D'ailleurs les cercles en faveur de l'union étaient forts, et c'est sur leur inspiration qu'en 1431 le basileus envoya une ambassade au nouveau pape Eugène IV. Les négociations avec le pontife étaient positives sur le papier: on convint d'un nouveau concile dans une ville italienne auquel le basileus aurait personnellement pris part; pendant ce temps-là, en son absence, Constantinople serait gouvernée à sa place par son frère Constantin, ce qui aurait aussi fait cesser les querelles en Morée où celui-ci et ses frères se contestaient le pouvoir. Mais de son côté le nouveau concile réuni à Bâle avait entrevu dans l'union projetée un excellent moyen pour faire triompher les thèses en faveur du concile sur celles en faveur du monarque-pontife, et avait en ce sens tenté d'imposer son autorité aux grecs en envoyant Jean de Raguse, Henry Menger et Simon Fréron pour le représenter à Constantinople ${ }^{3}$.

Que le pape Eugène IV ait été effectivement décidé à traduire dans la pratique son programme d'aides à Constantinople en les faisant coïncider avec la période où l'empereur et le patriarche grec étaient absents de la ville, retenus par un concile qui se tenait dans une ville de l'Adriatique 4 , ressort clairement des actes de son représentant à Bâle, le cardinal Giulano Cesarini qui présidait le concile lui-même ${ }^{5}$. Recevant

${ }^{3}$ Cf. C.-J. Hefele - H. Leclercq, Histoire des conciles, VII, II, Paris, 1916, p. 916.

4Cf. D. Caccamo, «Engenio IV e la crociata di Varna», Archivio della Società romana di storia patria, LXXIX, 1956, p. 37.

${ }^{5}$ Cf. P. Becker, Giuliano Cesarini, Kallmünz, 1935. 
les ambassadeurs grecs au concile le 19 juillet 1434, le cardinal Cesarini prononçait une harangue compliquée où la lutte contre les turcs était considérée comme le résultat et la conséquence nécessaire de l'unité religieuse obtenue ${ }^{6}$. Les intentions du cardinal Cesarini (et de Eugène IV) et celles des ambassadeurs conciliaires à Constantinople - qui étaient arrivés dans cette ville le 23 septembre 1435 et avaient presque immédiatement obtenu audience auprès de l'empereur - paraissaient coïncider. Il est vrai que Jean de Raguse, Menger et Fréron essayaient à tout prix de démontrer au basileus, au patriarche et aux principaux membres du parti en faveur de l'union que la ville de Bâle était celle convenant le mieux au déroulement du concile, et en échange n'obtenaient des grecs que leur renonciation à la proposition qu'ils avaient avancée avant de réunir le concile à Constantinople, mais accompagnée d'une ferme indication pour une ville maritime. Mais les graves conditions où se trouvaient les grecs et aussi les hongrois, tous deux exposés à la menace turque, impressionnaient si fort Jean de Raguse qu'il l'écrivit, le 9 février 1436, au concile et au cardinal Cesarini en fournissant d'ailleurs des informations reflétant bien le danger et la confusion qui régnaient à l'époque. Le bruit courait en effet que le sultan Murat était sur le point d'abandonner l'Europe, obligé d'accourir en Asie pour faire face aux menaces de l'empereur des "tartares» (c'est-à-dire, probablement, Ibrahim Beg, le seigneur turkmène de la Caramanie); mais il semblait par ailleurs que les ottomans se préparaient au contraire à lancer une autre attaque contre les hongrois, comme cela se produisit en effet; Jean de Raguse faisait enfin allusion à des discordes de nature religieuse qui avaient surgi entre les ottomans et étaient d'une si grave nature que les puissances chrétiennes unies, une fois l'accord conclu, auraient eu tout loisir de refouler les turcs très loin dans les terres en Asie Mineure ${ }^{7}$.

Mais c'était justement sur la croisade que le différent entre le pape et le concile se manifestait d'une manière de plus en plus claire et forte. D'une part, le premier tenait à faire savoir au basileus qu'il était le seul à avoir l'autorité, le prestige et le pouvoir nécessaires pour pouvoir mobiliser les puissances occidentales, mais qu'il n'aurait pu le faire si le Schisme d'Orient ne prenait pas fin auparavant; de l'autre, le second se présentait comme le seul lieu où aurait pu concrètement se réaliser non seulement l'union mais aussi la convergence des princes ou de leurs représentants accrédités pour programmer sérieusement une expédition militaire. Mais à faire pencher Jean VIII vers le pontife, dont les difficultés devaient tout de même être connues à Constantinople, convergeaient à la fois les nouvelles des différents et des litiges provenant continuellement de Bâle, et les deux points fermes du programme de la majorité conciliaire qui semblaient être faits exprès pour que les sympathies du basileus s'éloignent du concile: tout d'abord la question préjudicielle de la suprématie du concile sur le pape (sujet qui aurait à lui seul constitué un insurmontable obstacle à la négociation de tout autre thème) ; et puis l'inamovibilité du concile luimême de Bâle, siège que les grecs jugeaient tout à fait inadapté à leurs nécessités.

Avec 1437, l'équilibre des forces et le pouvoir respectif des deux partis ecclésiastiques devinrent plus clairs; d'une part, la majorité conciliaire de Bâle s'était déclarée en faveur du transfert du concile en Avignon et avait offert de mettre à la

${ }^{6} \mathrm{Cf}$. E. Cecconi, Studii storici sul concilio di Firenze, Firenze, 1869, I, n. 28.
${ }^{7}$ Caccamo, Eugenio $I V$, art. cit. 
disposition du basileus et du patriarche des bateaux et des moyens économiques pour le voyage en Occident ; mais elle avait ensuite prouvé n'être en mesure de traduire en pratique ni l'une ni l'autre de ces décisions. D'autre part, la minorité conciliaire, fidèle au pontife et à Cesarini, s'était prononcée pour le transfert du concile à Udine ou à Florence et semblait montrer des caractères de plus grande crédibilité. L'empereur Sigismond, qui s'était vu refuser son offre de transfert du concile à Buda, déconseillait au basileus de partir pour l'Europe, mais il était désormais au crépuscule de sa vie et il disparaîtra en effet en décembre pour laisser la place à Albert II d'Habsbourg, avec lequel le pape avait déjà pris des accords. Le sultan Murat suivait avec appréhension et méfiance l'évolution de la situation en laissant croire que l'éloignement de l'empereur de sa capitale aurait encouragé les ottomans à l'attaquer de nouveau et plus durement; en fait, le souverain turc était assez préoccupé du fait que la présence du basileus en Occident puisse vraiment déterminer une sérieuse expédition de croisés.

Le 18 octobre 1437 le pape émettait la bulle Doctoris gentium par laquelle répondant à l'acte de rupture définitif de la majorité conciliaire contre lui, le Monitorium du mois de juillet précédent - il dénonçait l'immobilisme et la stérilité des assises de Bâle et en décrétait le transfert à Ferrare. Centre de l'État d'Este allié aux vénitiens et aux florentins, cette ville d'Émilie - un des centres sur lesquels les grecs eux-mêmes avaient formulé un avis favorable, bien reliée comme elle l'était avec la mer grâce au delta du Po - présentait toutes les conditions requises pour satisfaire les parties. Eugène IV comptait sur l'appui financier de Florence, depuis peu tombée entre les mains de son ami, le «seigneur occulte» Côme de Médicis, et sur celui militaire et maritime de Venise. Par ailleurs florentins et vénitiens, alors en lutte contre le duc de Milan, Filippo Maria Visconti, avaient intérêt à maintenir le pontife de leur côté et savaient que la meilleure façon de poursuivre ce but était de le soutenir contre un concile comme celui de Bâle, désormais réduit à un rassemblement de bavards et de chicaneurs qui voyaient de jour en jour le prestige accumulé dans les années précédentes s'éloigner d'eux. L'étape à Ferrare était bien entendu prévue comme provisoire avant le transfert définitif du concile à Florence; mais elle était aussi nécessaire pour venir à l'encontre des désirs des grecs, et la nouvelle de l'évolution de la situation en ce sens était d'ailleurs déjà parvenue sur la Corne d'Or durant l'été 1437, et Jean de Raguse lui-même - qui se sentait désormais abandonné par les prélats réunis à Bâle - avait commencé à travailler sur la base de ce qu'il avait entendu dire.

Se suivant de près, les ambassadeurs et les modestes flottes envoyés respectivement par le pape et par la majorité du concile de Bâle arrivèrent en tout cas à Constantinople entre septembre et octobre 1437. Le pauvre mais raisonnable Jean de Raguse ne savait plus vraiment où donner de la tête ; quoi qu'il en soit, il passa de nouveau loyalement du côté de ce concile qu'il avait abandonné, convaincu de sa dissolution, en essayant de maintenir une position de médiateur. Mais les offres du pontife, qui s'appuyaient désormais sur la puissance des galères vénitiennes et l'or florentin - et les grecs étant, ainsi que Enea Silvio Piccolomini les décrivait, avec 
peu de mansuétude, comme étant "pauvres et très habiles à mendier»" - avaient convaincu le basileus et le patriarche. Jean VIII communiqua sa décision aux envoyés de Bâle en la motivant par le fait que le concile avait manqué à sa promesse d'envoyer à Constantinople les moyens nécessaires à son voyage et à la défense de la ville durant son absence. Il est vrai qu'une flotte conciliaire avait quand même fini par arriver et que le pape n'avait par ailleurs encore pourvu concrètement à la partie militaire de leurs accords ; néanmoins, ses offres paraissaient désormais plus crédibles et Jean de Raguse lui-même - qui, à ce sujet, envoyait aux prélats de Bâle un rapport qui fut lu au cours de la séance concilaire du 29 janvier $1439^{\circ}$ - n'avait rien pu opposer de sérieux aux raisons de l'empereur.

Après avoir quitté Constantinople à la fin du mois de novembre 1437 à bord des bateaux que le pontife avait mis à leur disposition, les représentants grecs au concile, sous la conduite du basileus et du patriarche, arrivèrent à Venise le 8 février $1438^{10}$.

Les grecs furent accueillis à Venise par Ambrogio Traversi, ami du pontife (il avait aussi été l'un des inspirateurs de la bulle dénonçant le concile de Bâle) et général de l'ordre de Camaldoli, qui se chargea de faire les honneurs de la maison et de remettre au basileus et au patriarche la somme d'argent nécessaire pour affronter les premiers frais. L'empereur quitta Venise le 28 février et le reste de la délégation, y compris le patriarche qui était en mauvais état de santé, le suivit quelques jours plus tard, car ils n'avaient, paraît-il, pas suffisamment d'embarcations à leurs disposition. Le basileus arriva à Ferrare le 4 mars et les autres le suivirent trois jours plus tard: entre temps les ambassadeurs du pontife à Constantinople, Nicola di Cusa et les évêques du Portugal et de Digne, présentaient au pape un rapport sur leur mission.

Les travaux à Ferrare se déroulèrent au milieu de nombreuses questions d'étiquette et de procédure car, dans la mesure où ils se sentaient justement plus faibles par rapport aux latins, les grecs étaient extrêmement susceptibles en matière de préséance et d'actes ou de symboles de suprématie. D'autres débours d'argent furent nécessaires pour étouffer telle ou telle remontrance. Enfin - malgré les foudres que les prélats encore réunis à Bâle lançaient contre le «conciliabule» de Ferrare - les travaux commencèrent le 9 avril en la présence du basileus et des délégués des patriarches d'Alexandrie, d'Antioche et de Jérusalem. De son côté Joseph, le patriarche de Constantinople, ne put assister à l'inauguration solennelle à cause de son infirmité, mais il envoya un message de bienvenue au concile.

Tandis que se déroulaient les travaux de la commission paritaire latino-grecque visant à aplanir les points de friction bien connus entre les deux Églises (la procession du Saint-Esprit, la question des azymes, la doctrine du Purgatoire, le problème de la primauté entre les sièges des patriarches), le basileus ne manquait pas de manifester son impatience à l'égard de la fraction des prélats grecs moins favorables à l'union - fraction guidée par Marc, évêque d'Ephèse - et des discussions théologi-

\footnotetext{
${ }^{8}$ Enea Silvio Piccolomini papa Pio II, I commentarii, I, 7, ed. L. Totaro, Milano, 1984, pp. 30-31.

${ }^{9}$ Caccamo, Eugenio IV, p. 39.

${ }^{10} \mathrm{Cf}$. Hefele-Leclercq, Histoire des conciles, VII.II, op. cit., p. 960 sgg.
} 
ques trop poussées. Il était également hostile à ce que les travaux avancent trop rapidement, car il espérait que les prélats de Bâle s'uniraient eux aussi au concile de Ferrare pour lui conférer un plus grand prestige et, surtout, que les ambassadeurs des puissances occidentales fussent arrivés. D'ailleurs, ce qui lui importait était d'arriver rapidement à une solution en faveur de l'union, quelle qu'elle soit, préférant probablement qu'elle restât vague, pour lui permettre de rentrer à Constantinople et imposer en même temps à ce moment-là le prix de l'union, à savoir l'expédition militaire pour défendre son empire ou ce qui en restait. Sa principale activité pendant son séjour à Ferrare fut en tout cas de chasser sur les réserves du marquis Niccolò III. En fait, entre le basileus, le pape et les prélats grecs plus hostiles à la thèse de l'union, s'instaurait peu à peu une escarmouche faite de réticences réciproques; Jean ne voulait pas que ses hommes s'engageassent dans des discussions théologiques trop subtiles qui auraient pu retarder l'union; mais il ne voulait en même temps pas y arriver trop facilement et sans avoir auparavant reçu des garanties précises sur les aides militaires; Eugène entendait au contraire l'union comme condition sine qua non pour résoudre sérieusement le problème que constituait l'expédition occidentale au secours de Constantinople; les prélats grecs moins favorables à l'union faisaient n'importe quoi pour la retarder (certains essayèrent même de s'enfuir de Ferrare) en se justifiant auprès de l'empereur, irrité par leurs ratiocinations, par le fait - à vrai dire non sans fondement - qu'ils soupçonnaient en réalité le pape de ne pas avoir l'autorité nécessaire pour faire bouger les latins au secours de Byzance.

De son côté, Eugène IV faisait tout ce qu'il pouvait, en essayant de convaincre les princes occidentaux de prendre des engagements concrets, et en récoltant en même temps des fonds pour la défense de Constantinople ${ }^{11}$. La nouvelle nous est aussi parvenue d'une de ses lettres écrite au "Prêtre Gianni», c'est-à-dire au négus d'Éthiopie, lettre qu'il avait justement envoyée de Ferrare ${ }^{12}$.

Avec la première session du concile de Ferrare, le 8 octobre suivant, les récriminations reprirent. Les grecs étaient mécontents parce que le pontife, souffrant d'un accès de goutte, avait évité de tenir la session dans la cathédrale, pour lui trop éloignée; le basileus était en colère parce qu'on ne lui avait pas consenti d'arriver à cheval à la place qu'on lui avait assignée. Ces difficultés une fois aplanies, les travaux continuèrent sans que l'on abordât cependant la question de la croisade. Les ambassadeurs du duc de Bourgogne arrivèrent finalement au concile à la fin seulement du mois de novembre ; mais le basileus qui les attendait impatiemment fut déçu de leur comportement peu obséquieux à son égard et par le fait que dans le long message oral prononcé par l'abbé général de Cîteaux au nom de son seigneur l'on parlait beaucoup de l'union et de la réconciliation avec les prélats restés à Bâle, mais on ne disait rien des aides occidentales à Constantinople. Entre temps, les instances florentines pour le transfert du concile se faisait sentir de plus en plus fort; les grecs

\footnotetext{
${ }^{11}$ Caccamo, Eugenio $I V$, art. cit., pp. 39-40, G. Fedalto, Le Chiese d'Oriente, Da Giustiniano alla caduta di Costantinopoli, Milano, 1984, pp. 211-12.

${ }^{12}$ Cf. A. Frizzi, Memorie per la storia di Ferrara, III, Ferrara, 1850, pp. 479-80 ; G. Pistarino, «I portoghesi verso l'Asia del Prete Gianni», Studi medievali, s. III, vol. II, 1961, pp. 77137.
} 


\section{Franco CARDINI}

étaient à ce propos réticents mais, au début de janvier 1439, une bonne somme d'argent versée pour leurs frais supplémentaires et une autre, considérable, expédiée à Constantinople pour contribuer à la défense de la ville, permirent de les convaincre. Et comme ni les prélats de Bâle ni les puissances occidentales ne bougeaient, le basileus décida au moins d'exploiter les moyens financiers mis à sa disposition et de poursuivre depuis Florence son action pour convaincre les occidentaux de la nécessité de l'aider à repousser les ottomans ${ }^{13}$. L'union éphémère des deux Églises - le schisme renouvelé en Occident - et la malheureuse et tardive campagne de croisade qui s'en suivit et se termina en 1444 par le désastre de Varna, sont toutes choses connues.

Franco Cardini

${ }^{13}$ J. Gill, Il Concilio di Firenze, tr. it., Firenze, 1967. 